\title{
Performance following rewarded and nonrewarded trials at 12-min and 20-sec intertrial intervals ${ }^{1}$
}

Two groups of rats were given $50 \%$ partial reinforcement for 192 trials in a straight alley, eight trials a day. For one group the intertrial interval was $12 \mathrm{~min}$, for the other, $20 \mathrm{sec}$. Faster performance was found on trials following reward throughout training at a 20-sec intertrial interval, but this effect was not observed at a 12-min intertrial interval.

Performance on the early trials of partial reward (PR) training is often superior on trials following reward (TFR) relative to trials following nonreward (TFN) (e.g., Bloom, 1967; McCain, 1966). Both Bloom and McCain have suggested that this finding is indicative of the presence of stimulus aftereffects which could be conditioned to the response by reinforcement on succeeding trials, as proposed by the revised aftereffects hypothesis (Capaldi, 1966).

There are two problems to which studies demonstrating superior TFR performance have not so far addressed themselves. First, no specific inquiry has been made into the duration of differential performance over a considerable number of training trials, and it seems to have been tacitly assumed that the effect is transient and disappears relatively early in PR acquisition. Second, the TFR effect has not been investigated systematically at different intertrial intervals (ITIs). It would seem reasonable to suppose that if, as McCain (1966) has suggested, increased vigor on TFRs is a motivational phenomenon, such motivational effects might be expected to disappear or dissipate over time. We report two experiments designed to provide some information on these questions.

\section{Experiment 1} METHOD

Ten male Wistar strain albino rats, 60-70 days old, were maintained on a $23 \mathrm{~h} 10 \mathrm{~g}$ food deprivation schedule for 21 days prior to experimental training, during which time they were handled and tamed.

The apparatus has been described elsewhere (Surridge \& Amsel, 1966). Briefly, a gray entry box and start box were connected in series to a black $31 / 4-$ $\mathrm{ft}$ wooden runway which provided three 1-ft time measures of locomotor performance.

Ss were given a $50 \%$ PR schedule for 192 trials, eight trials a day, at a 12-min ITI. Reward was one $250 \mathrm{mg}$ Noyes pellet. Ss were detained in the goal box $30-35 \mathrm{sec}$ on every trial. Following training they were returned to their home cages and given the daily food ration 30-40 min later.

The daily sequences of eight trials contained four re- warded (R) and four nonrewarded (N) trials, there being eight such sequences in all. Within each block of four days there were two Ns and two Rs in each trial position; there were equal numbers of TFR and TFN; it was equiprobable that any trial would be followed by an $\mathrm{N}$ or $\mathrm{R}$ trial; and there was neither single altermation nor a block of more than three trials of the same type. The daily sequences were randomized across Ss so that as far as possible no two Ss received the same order on any one day.

\section{Experiment 2}

The Ss were seven male Wistar strain albino rats, 60-70 days old. Habituation, apparatus, and experimental procedure were the same as in Experiment 1. The only difference was that Ss were run their eight daily trials at a 20-sec ITI.

\section{RESULTS AND DISCUSSION}

Reciprocals were taken of the raw scores from each experiment, converting them to speeds (ft/sec). Figure 1 plots, for both experiments, mean speeds on TFRs and TFNs in each runway measure against the six 4-day blocks. Each point represents the mean of the 14 TFRs or TFNs within a 4-day block.

There was no consistent tendency in Experiment 1 for higher speeds on either TFRs or TFNs. Analyses of variance comparing TFR and TFN performanceover the six four-day blocks yielded a nonsignificant main effect ( $p>.05$ in all measures). The TFR-TFN by Day Block interaction reached a marginal level of significance in the start measure $(F=2.75, \mathrm{df}=5 / 45$, $p<.05$ ), but was not significant in either the run or the goal measure $(p>.05)$.

In Experiment 2, Ss ran faster on their TFRs throughout the entire course of training, and the effect was most pronounced in the goal measure. Analyses of variance confirmed this result: The TFRTFN difference was not significant in the start measure $(p>.05)$, but was significant in the run measure $(F=6.18$, df $=1 / 30, p<.025)$, and in the goal measure $(\mathrm{F}=22.89, \mathrm{df}=1 / 30, \mathrm{p}<.001)$. The $\mathrm{TFR}-\mathrm{T} F \mathrm{~N}$ by Day Block interaction was not significant in any measure ( $p>05$ in all cases). The reduced TFR-T FN difference in the start measure probably reflects the operation of the frustration effect (FE) (Amsel \& Roussel, 1952), which has its effect most strongly at the beginning of the response chain. Though the FE would probably have dissipated somewhat during the 30-35 sec goal confinement and the 20-sec ITI, it seems conceivable 




Fig. 1. Mean speeds in Experiments 1 and 2 on TFRs and TFNs on each of the six 4-day blocks. that increased vigor on a TFN due to frustration could have counteracted the opposite tendency on a TFR in the start measure.

The evidence from our two experiments suggests that some form of stimulus trace produced by the goal event on one trial remains operative on the next trial when highly massed trials are employed, but that the trace has dissipated by the time 12 min have elapsed. Also, the fact that faster TFR performance was observed in our 20-sec ITI condition throughout the entire $192 \mathrm{ac}-$ quisition trials is one which suggests that the phenomenon is not necessarily transient.

Our data add support to other experimental evidence which indicates that at short trial spacing the stimuli produced by reward and nonreward do carry over from one trial to the next and affect performance in differential ways. McCain (1966) has demonstrated faster T FR performance under certain experimental conditions employing ITIs of approximately $20 \mathrm{sec}$ with as few as three or four acquisition trials, which would suggest an immediate carried-over effect rather than the development of some form of associative mechanism. A study by Surridge, Rashotte \& Amsel (1967), which employed four partially rewarded acquisition trials and which was run at a 20-sec ITI also showed the faster TFR performance; however, some recently collected and as yet unpublished data from the Toronto laboratories, run under essentially similar conditions to those of Surridge, Rashotte and Amsel, but employing a 24-h ITI failed to show faster performance on TFRs. Bloom (1967), using a 20-sec ITI found that the $T F R$ phenomenon varied in strength with the magnitude of reward and also with the goal box detention time on $N$ trials; "running time was fastest following large reward, slower following small reward, slower still following short nonreward, and slowest following long nonreward" (p. 38). Bloom concluded that this performance gradient indicated differences in unconditioned response strength for performance after different amounts of reward and nonreward.

At very short trial spacing, then, it would seem difficult in PR experiments to be sure that successive trials are entirely discrete from one another, even when a different stimulus complex is presented between trials. This would seem to hold particular relevance for PR experiments in either the operant conditioning mode or in runways in which multiple trials are run each day under highly massed conditions, since conclusions drawn from such experiments may in some ways be inapplicable to PR experiments run at longer trial spacing, when presumably the aftereffect has had time to dissipate.

\section{References}

AMSEL, A., \& ROUSSEL, J. Motivational properties of frustration: I. Effect on a running response of the addition of frustration to the motivational complex. J. exp. Psychol., 1952, 43, 363-368.

BLOOM, J. M. Early acquisition responding on trials following different rewards and nonrewards. Psychon Sci, 1967, 7, 37-38.

CAPALDI, E. J. Partial reinforcement: An hypothesis of sequential effects. Psychol. Rev., 1966, 73, 459-477.

McCAIN, G. Partial reinforcement effects following a small number of acquisition trials. Psychon. Monogr. Supp., 1966, 1, 252-270.

SURRIDGE, C. T., \& AMSEL, A. Acquisition and extinction under single alternation and random partial reinforcement conditions with a 24hour intertrial interval. J. exp. Psychol., 1966, 72, 361-368.

SURRIDGE, C. T., RASHOTTE, M. E., \& AMSEL, A. Resistance to extinction of a running response after a small number of partially rewarded trials. Psychon. Sci, 1967, 7, 31-32.

Note

1. Supported by research Grants GB-3772 from the National Science Foundation and APB-72 from the National Research Council of Canada awarded to Professor Abram Amsel. 